\title{
Malaria prevention reduces in-hospital mortality among severely ill tuberculosis patients: a three- step intervention in Bissau, Guinea-Bissau
}

\author{
Raffaella Colombatti ${ }^{1,2 *}$, Martina Penazzato ${ }^{3}$, Federica Bassani ${ }^{2}$, Cesaltina Silva Vieira ${ }^{2}$, Antonia Araujo Lourenço ${ }^{2}$,
} Fina Vieira ${ }^{2}$, Simone Teso ${ }^{4}$, Carlo Giaquinto ${ }^{3}$, Fabio Riccardi ${ }^{2,5}$

\begin{abstract}
Background: Malaria and Tuberculosis (TB) are important causes of morbidity and mortality in Africa. Malaria prevention reduces mortality among HIV patients, pregnant women and children, but its role in TB patients is not clear. In the TB National Reference Center in Guinea-Bissau, admitted patients are in severe clinical conditions and mortality during the rainy season is high. We performed a three-step malaria prevention program to reduce mortality in TB patients during the rainy season.

Methods: Since 2005 Permethrin treated bed nets were given to every patient. Since 2006 environmental prevention with permethrin derivates was performed both indoor and outdoor during the rainy season. In 2007 cotrimoxazole prophylaxis was added during the rainy season. Care was without charge; health education on malaria prevention was performed weekly. Primary outcomes were death, discharge, drop-out.

Results: 427, 346, 549 patients were admitted in 2005, 2006, 2007, respectively. Mortality dropped from 26.46\% in 2005 to $18.76 \%$ in 2007 ( $p$-value 0.003 ), due to the significant reduction in rainy season mortality (death/discharge ratio: $0.79,0.55$ and 0.26 in 2005, 2006 and 2007 respectively; $p$-value 0.001) while dry season mortality remained constant (0.39, 0.37 and 0.32 ; $p$-value 0.647$)$. Costs of malaria prevention were limited: $2 € /$ person. No drop-outs were observed. Health education attendance was 96-99\%.

Conclusions: Malaria prevention in African tertiary care hospitals seems feasible with limited costs. Vector control, personal protection and cotrimoxazole prophylaxis seem to reduce mortality in severely ill TB patients. Prospective randomized trials are needed to confirm our findings in similar settings.
\end{abstract}

Trial registration number: Current Controlled Trials: ISRCTN83944306

\section{Background}

Malaria and Tuberculosis are important causes of morbidity and mortality in Africa [1] and are among the main reasons of hospital admission and in-hospital mortality [2-5]. During the rainy season, malaria burden increases [6-8] in many African countries, peaking morbidity and mortality. Regardless of HIV status, malaria infection affects severely ill TB patients who are already compromised by malnutrition, deprived immunity or disseminated disease $[6,9,10]$. In fact, in pulmonary TB

\footnotetext{
* Correspondence: rcolombatti@gmail.com

'Clinic of Pediatric Hematology-Oncology, Department of Pediatrics,

University of Padova, Padova, Italy

Full list of author information is available at the end of the article
}

there is a transient systemic immunosuppression due to over expression of transforming growth factor beta and interleukin-10 [11]. Interactions between $\mathrm{TB}$ and malaria have been demonstrated both in vitro and in vivo: Plasmodium Falciparum modulates Mycobacterium Tuberculosis infection [12] and malaria has been shown to exacerbate mycobacterial infection [13]. The reasons for this are not completely explored but seem to involve parasite-parasite interaction and host-parasite interaction [12-14]: malaria causes a further depression in immunity through a qualitative and quantitative defect in $\mathrm{T}$ lymphocytes, mainly the CD8+ that are necessary for anti-mycobacterial response, and through a deregulation of the cytokine cascade. Moreover, the respiratory

\section{Biomed Central}


distress that is frequent during acute malaria both in children (due to metabolic acidosis) and adults (due to pulmonary edema and Acute Respiratory Distress Syndrome) [15], can worsen the respiratory effort related to TB. Therefore, given the multiple interactions between malaria and TB and the mutual effect in increasing mortality $[1,5,6]$, new strategies of integrated management should be investigated, especially during malaria peak transmission seasons. The identification of efficacious and low cost methods for reducing malaria in TB patients could largely improve clinical outcome and public health strategies optimizing the use of current limited resources.

Malaria prevention can be performed at various levels and several measures are proven to be quite effective in different groups of patients [16-20].

TB Reference Hospitals can be the sites to test integrated models of malaria prevention for severely ill patients due to the fact that those who are in poor conditions are generally admitted for at least the intensive phase of TB treatment (two months).

In the Hospital Raoul Follereau (HRF), National Reference Hospital for Tuberculosis and Lung Disease in Bissau, Guinea-Bissau, mortality during 2004 rainy season reached peaks of $60 \%$ in admitted patients. According to the clinical reports made by the hospital's physicians, high fever with Plasmodium Falciparum positive blood film was the main reason of death. In order to reduce mortality during the rainy season in admitted TB patients, since January 2005 we performed a malaria prevention program in the hospital. Our hypothesis was that the successive combination of personal protection, vector control and cotrimoxazole prophylaxis could be progressively more effective in reducing mortality in severely affected TB patients, who are admitted for long periods of time. Several other drugs with a potential better efficacy are used for malaria prophylaxis, but in the peculiar context of ТВ Units where almost half of the patients is HIV $+[1,21,22]$, the use of cotrimoxazole would potentially integrate the interventions and facilitate the long-term administration by the health care workers.

\section{Methods \\ Setting}

Guinea-Bissau is located in the West African Atlantic Coast. There are two climatic seasons: a rainy season from July to October and a dry season from November to June. Malaria is endemic with a peak transmission during the rainy season. The Hospital Raoul Follereau (HRF) is the National Reference Hospital for Tuberculosis and Lung Disease in Bissau, capital of Guinea-Bissau. According to the National Guidelines for TB, TB patients in poor clinical conditions or with severe disease (i.e important wasting or respiratory distress) are admitted after referral from regional hospitals or from TB health centers across the entire country.

After being destroyed during the 1998-1999 civil war, the hospital was rebuilt and reopened to the public in 2004. The hospital is a $2500 \mathrm{sqm}$ structure surrounded by a $7500 \mathrm{sqm}$ grass garden (Figure 1). The whole facility includes an in-patient service (three wards: men, women and children), an outpatient service, a laboratory, two X-Ray Units, a pharmacy, a service area (kitchen, laundry, ironing) and two cafeterias. From 2003 to 2008 the hospital policy was to perform clinical evaluation, diagnostic tests, treatment and nutritional support without charge to all admitted patients.

\section{Study outline and Malaria Prevention}

Malaria prevention was performed through a three-step intervention. A Permethrin treated bed net was given to every patient upon admission since 2005 and during all the three years of the intervention; bed nets were retreated every 30-45 days.

Environmental disinfection was performed monthly with Permethrin based products by appropriately trained hospital staff according to manufactory's instructions during the rainy season of 2006 and 2007; spraying was conducted both outdoor (with Microsin) and indoor (with Petrin L) on doors and window frames. The only difference was that in 2006 environmental outdoor and indoor disinfection was repeated monthly from July 15th to October 30th while in 2007, due to increased availability of the products, it was repeated monthly from June 1st to October 30th.

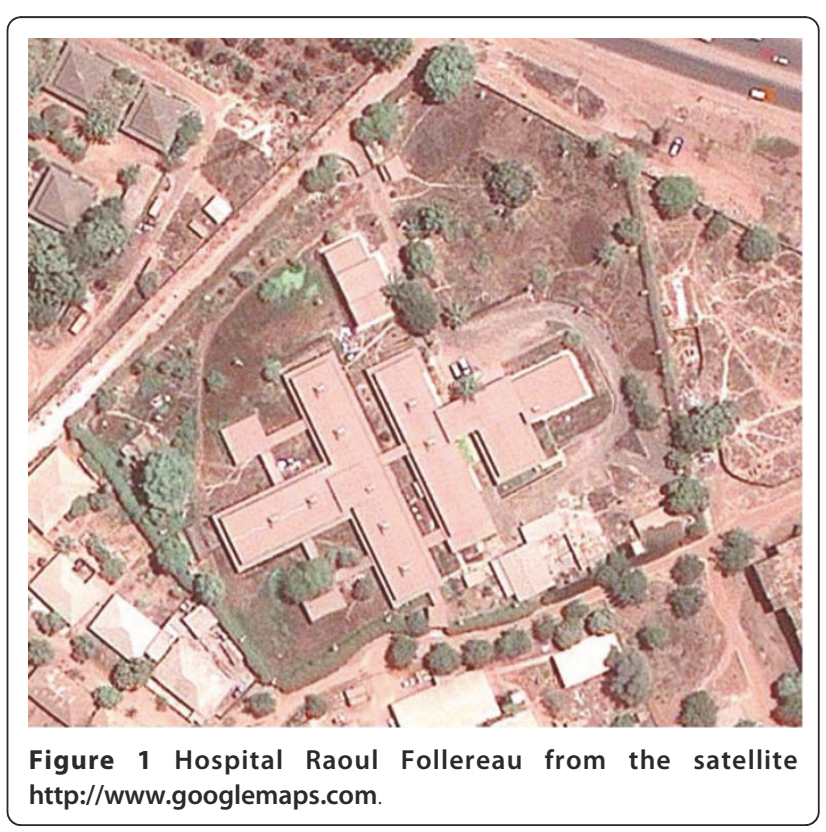


Prophylaxis with once daily Trimethoprim $80 \mathrm{mg} / \mathrm{Sul}-$ famethoxazole $400 \mathrm{mg}$ tablet was given to every patient admitted from July 1st to October 30th only in 2007.

The study was approved by the Hospital Board. All patients gave informed oral consent and all patients benefited from the interventions.

\section{Diagnosis of TB and Malaria and standard treatment}

As part of routine clinical evaluation, patients who were admitted with a suspect of $\mathrm{TB}$, received a three sample sputum analysis, a thorax X-Ray, a complete blood count, a biochemistry analysis and a malaria thick film. Ziehl-Neelsen's sputum staining technique was used and patients were considered smear positive if acid fast bacilli were shown on at least two samples. Smear negative patients were considered to have TB according to the physician's evaluation of chest X-Ray and clinical condition. Additional analyses were performed if necessary, based on physician's judgment.

HIV testing and Antiretroviral treatment were offered free of charge to patients admitted from July to August, in November and in March of every year; alternatively, due to the limited availability of free reagents and drugs, patients were advised to undergo HIV testing in other health facilities and return back the result.

A clinical episode of malaria was defined as an axillary temperature $\geq 37.5^{\circ} \mathrm{C}$ together with the presence of malaria parasitemia at any density.

The hospital treatment protocols for TB, Malaria, HIV/AIDS and other diseases were the same in 2005, 2006, 2007. TB was treated according to the National Guidelines, with a four drug regimen for two months (rifampicin, isoniazid, ethambutol and pyrazinamide) followed by two drugs for the following six months (isoniazid and ethambutol); five drugs were used in case of relapsed TB (streptomycin, rifampicin, isoniazid, ethambutol and pyrazinamide). Chloroquine was administered as standard treatment in case of uncomplicated malaria, while severe malaria was treated with iv Quinine, according to the National Program against Malaria in Guinea-Bissau. Other medications were given on a clinical basis. Nutritional support was similarly implemented over the three years and included three main meals and two snacks given daily to all patients.

Compliance to treatment (TB drugs, cotrimoxazole and other drugs) was assured by direct observation of drug intake by the nurses.

Health education on TB, malaria, HIV, water-borne diseases and personal hygiene was part of the hospital care standards: every week an appropriately trained nurse performed health education lessons to in-patients and the number of participants was recorded. The importance of malaria prevention and bed net use was particularly stressed during the lessons.

\section{Outcomes}

Primary outcomes were death or discharge from hospital. Secondary outcomes were drop-outs (defined as voluntary discharge from hospital before recovery against physician's advice and/or treatment abandonment) and percentage of attendance of health education classes.

\section{Data collection}

Personal and clinical information were transferred from patient's individual charts to Microsoft Access 2000 (Microsoft Corporation, Seattle, WA, USA). Hospital registries were used to cross-check diagnosis and admission-discharge dates. Pearson's chi-square test was used to compare variables within groups. $p$-values $<0.05$ were considered statistically significant.

\section{Results}

A total of 427, 346 and 549 patients were admitted in 2005, 2006 and 2007, respectively. Major baseline characteristics -gender, symptoms on admission and diagnosis- were similar in the three groups of patients as shown in Table 1 . The majority of patients were adults ranging from 15 to 59 years, even if the +60 years group was less numerous in 2006. TB diagnosis was confirmed

Table 1 Patient's clinical characteristics

\begin{tabular}{|c|c|c|c|c|c|c|c|}
\hline \multirow{3}{*}{ Admissions $\left(\mathrm{N}^{\circ}\right)$} & \multicolumn{2}{|c|}{2005} & \multicolumn{2}{|c|}{2006} & \multicolumn{2}{|c|}{2007} & \multirow[t]{2}{*}{$p$-value } \\
\hline & \multicolumn{2}{|c|}{427} & \multicolumn{2}{|c|}{346} & \multicolumn{2}{|c|}{549} & \\
\hline & $N^{\circ}$ & $\%$ & $N^{\circ}$ & $\%$ & $N^{\circ}$ & $\%$ & \\
\hline \multicolumn{8}{|l|}{ Gender } \\
\hline M & 232 & $54.3 \%$ & 203 & $58.8 \%$ & 310 & $56.4 \%$ & 0.480 \\
\hline $\mathrm{F}$ & 195 & $45.7 \%$ & 143 & $41.2 \%$ & 239 & $43.6 \%$ & \\
\hline \multicolumn{8}{|l|}{ Age (years) } \\
\hline $14-$ & 53 & $12.4 \%$ & 55 & $15.9 \%$ & 92 & $16.8 \%$ & \\
\hline $15-59$ & 325 & $76.1 \%$ & 268 & $77.5 \%$ & 395 & $71.9 \%$ & 0.043 \\
\hline $60+$ & 49 & $11.5 \%$ & 23 & $6.6 \%$ & 62 & $11.3 \%$ & \\
\hline \multicolumn{8}{|l|}{$\begin{array}{l}\text { Symptoms on } \\
\text { admission }\end{array}$} \\
\hline Cough & 392 & $92 \%$ & 317 & $91.6 \%$ & 499 & $90.8 \%$ & \\
\hline Fever & 366 & $85.7 \%$ & 294 & $84.9 \%$ & 486 & $88.5 \%$ & 0.866 \\
\hline Chest pain & 319 & $74.7 \%$ & 252 & $72.8 \%$ & 428 & $78 \%$ & \\
\hline Weight loss & 289 & $67.7 \%$ & 248 & $71.6 \%$ & 390 & $71 \%$ & \\
\hline \multicolumn{8}{|l|}{ Diagnosis } \\
\hline TB & 306 & $71.7 \%$ & 239 & $69.1 \%$ & 390 & $71 \%$ & \\
\hline $\begin{array}{l}\text { Pulmonary TB } \\
\text { Smeart }\end{array}$ & 157 & & 120 & & 195 & & \\
\hline $\begin{array}{l}\text { Pulmonary TB } \\
\text { Smear- }\end{array}$ & 107 & & 99 & & 150 & & 0.387 \\
\hline $\begin{array}{l}\text { Extra Pulmonary } \\
\text { TB }\end{array}$ & 32 & & 20 & & 31 & & \\
\hline Lung Disease No TB & 94 & $22 \%$ & 83 & $23.9 \%$ & 110 & $20 \%$ & \\
\hline Other & 27 & $6.3 \%$ & 24 & $7 \%$ & 49 & $9 \%$ & \\
\hline
\end{tabular}


in most patients (69.1-71.7\%) with smear positive TB being more frequent. HIV prevalence was similar (43\%, $45 \%$ and $48 \%$ in 2005, 2006 and 2007, respectively) as well as the proportion of patients receiving Antiretroviral Therapy every year (78\%, 75\%, 79\% in 2005, 2006 and 2007 respectively). Between 64-69.8\% were HIV1, 20-23.5\% were HIV2 and 10.2-11.7\% HIV1/2.

Mean Length of Stay (LOS) during the three years was 78 days $(77,87,70$ days in 2005, 2006, 2007 respectively).

\section{Outcomes}

A significant reduction in mortality was observed from 2005 to 2007 (26.46\% vs $18.76 \%$, p-value 0.003). Detailed outcomes for rainy season and dry season are presented in Table 2 and Table 3 respectively. The main determinant of the annual mortality reduction was shown to be the drop of rainy season mortality. In fact, the death/discharge ratio significantly decreased during the rainy season (0.79, 0.55 and 0.26 in 2005, 2006 and 2007 respectively; p-value 0.001$)$ while remained fairly stable during the dry season (0.39, 0.37 and 0.32 in 2005, 2006 and 2007 respectively; p-value 0.647 ).

Deaths occurring during the first week of admission and therefore not influenced by malaria prophylaxis, were $41 \%$, $28 \%$ and $47 \%$ during the rainy season in 2005, 2006 and 2007 respectively ( $\mathrm{p}$-value 0.178 ). Death/Discharge ratio calculated considering only deaths occurring after the first week, were again highly significant (p-value 0.001): 0.51, 0.40 and 0.14 in 2005, 2006 and 2007 respectively.

None of the patients abandoned treatment or asked to be discharged before completing the two months of TB intensive phase treatment or before clinical recovery.

Every health education class was attended by $96-99 \%$ of the admitted patients.

A reduction in Plasmodium Falciparum positive blood films was observed from the period August-November 2005 to August-November 2006 and 2007 (23.65\% vs.
$15.7 \%$ vs. $8.4 \%$ respectively, p-value 0.001 ) but the results were not registered on a regular basis.

\section{Costs}

The overall cost of the entire malaria prevention program in 2007 was estimated to be $€ 1050$ (USD 1402). This figure includes: re-treatment of bed nets every 45 days along the year, outdoor and indoor disinfection performed during the rainy season and cotrimoxazole prophylaxis during the rainy season, with a mean cost of $2 € /$ patient. Additional purchases were the spray pump and the facial mask that can be used for several years ( $€ 500$ or USD 668). Bed nets were donated to the hospital by the Global Fund Malaria initiative over the three years.

\section{Discussion}

Our study suggests that malaria prevention is feasible in African hospital settings and can involve both environment-focused and patient-focused interventions. Permethrin treated bed nets, outdoor and indoor environment disinfection and cotrimoxazole prophylaxis seem to have a cumulative protective effect on survival in severely ill TB patients.

Malaria and TB are a heavy burden to African health systems; the arrangement of integrated approaches of management with limited resources is still a challenge $[1,5,6]$. Seasonal variation in malaria related mortality $[7,23]$ is well known in West-African countries and seasonal interventions have been effective in Gambia, Guinea-Bissau and Senegal [7,18,24]. Guinea-Bissau has one of the highest incidences of TB in the world [1] and malaria is an important death determinant in the country, since $45 \%$ of registered deaths are caused by malaria $[25,26]$. The majority of malaria related deaths occurs during the rainy season $[25,26]$. Therefore, it seems reasonable to target malaria prevention strategies to the rainy season.

Table 2 Rainy season (July-October) outcomes of hospitalization in 2005, 2006 and 2007

\begin{tabular}{|c|c|c|c|c|}
\hline Outcomes & 2005 & 2006 & 2007 & p-value \\
\hline \multicolumn{5}{|l|}{ Admissions } \\
\hline Total $\left(n^{\circ}\right)$ & 158 & 130 & 173 & \\
\hline Mean per month & 39,5 & 32,5 & 43,25 & \\
\hline \multicolumn{5}{|l|}{ Deaths } \\
\hline Total $\left(n^{\circ}\right)$ & 60 & 44 & 36 & \\
\hline Mean per month & 15 & 11 & 9 & \\
\hline \multicolumn{5}{|l|}{ Discharges } \\
\hline Total $\left(n^{\circ}\right)$ & 76 & 80 & 137 & \\
\hline Mean per month & 19 & 20 & 34.25 & \\
\hline \multirow[t]{2}{*}{ Mortality (Death/Admission)\% } & 37.9 & 33.8 & 20.8 & 0.0032 \\
\hline & (Cl 95\%:30.4\%-45.54\%) & (Cl 95\%:25.72\%-41.98\%) & (Cl 95\%:14.76\%-26.86\%) & \\
\hline Death/Discharge ratio & 0.78 & 0.55 & 0.26 & 0.001 \\
\hline
\end{tabular}


Table 3 Dry season (November-June) outcomes of hospitalization in 2005, 2006, 2007

\begin{tabular}{|c|c|c|c|c|}
\hline Outcomes & 2005 & 2006 & 2007 & p-value \\
\hline \multicolumn{5}{|l|}{ Admissions } \\
\hline Total $\left(n^{\circ}\right)$ & 269 & 216 & 376 & \\
\hline Mean per month & 33.62 & 27 & 47 & \\
\hline \multicolumn{5}{|l|}{ Deaths } \\
\hline Total $\left(n^{\circ}\right)$ & 53 & 51 & 67 & \\
\hline Mean per month & 6.62 & 6,37 & 8,37 & \\
\hline \multicolumn{5}{|l|}{ Discharges } \\
\hline Total $\left(n^{\circ}\right)$ & 137 & 136 & 208 & \\
\hline Mean per month & 17.12 & 17 & 26 & \\
\hline \multirow[t]{2}{*}{ Mortality (Death/Admission)\% } & 19.7 & 23.6 & 17.8 & 0.385 \\
\hline & (Cl 95\%: 14.95\%-24.45\%) & (CI 95\%:17.95\%-29.27\%) & (Cl 95\%:13.95\%-21.69\%) & \\
\hline Death/Discharge ratio & 0.38 & 0.37 & 0.32 & 0.647 \\
\hline
\end{tabular}

All the three interventions seem to produce a certain benefit, even if environmental disinfection and cotrimoxazole prophylaxis seemed to be the most effective.

Permethrin treated bed nets reduce malaria infection offering both a mechanical and chemical barrier to mosquito bites and are efficacious in children and adults [16-18]. Insecticide treated bed nets and cotrimoxazole prophylaxis have shown to reduce malaria infection in HIV+ adults [19,27], children [28] and pregnant women $[20,29,30]$. Environmental disinfection, with indoor and outdoor spraying, is a useful tool to fight malaria in several countries [21,31,32]. A limit of vector control strategies has been the difficulty to deliver the reagents on time (i.e. at the beginning of the rainy season) to households spread in large areas and to perform re-treatment on schedule [31]. A hospital-based intervention overcomes these problems since patients are admitted in the same place for several months. Hospital outdoor and indoor spraying leads to a parasite intensity/density reduction and therefore can be an additional strategy to reduce the malaria burden in severely ill TB patients $[20,32]$.

Cotrimoxazole prophylaxis administered to HIV+ patients has reduced malaria episodes in their HIV-seronegative household members [33] and was also efficacious in Mali with $99 \%$ prophylactic efficacy against malaria infection and disease in children [34]. Limited information are available on the role of cotrimoxazole for malaria prophylaxis in HIV-seronegative TB patients, even if once daily cotrimoxazole has reduced overall mortality in TB patients regardless of HIV status in South Africa [21] and also mortality and malaria infection in HIV+ TB patients in Abidjan [22].

The benefit of cotrimoxazole in our study may have been enhanced by the potential reduction of gastrointestinal illnesses, sepsis $[34,35]$, pneumonia and toxoplasmosis [36,37], often leading causes of death in immunocompromised patients. These factors have not been directly investigated by our study and a prospective randomized study may address the specific correlations. Nevertheless, the poor clinical conditions of in-patients with severe or disseminated TB infection, usually malnourished, might justify the use of cotrimoxazole for short periods of time, regardless their HIV status.

Concerns regarding the induction of high resistance to cotrimoxazole in non-typhoid salmonella bacteria or in other opportunistic pathogens and the implications on the potential benefits of co-trimoxazole prophylaxis in HIV-infected individuals have been raised; however, the size of this phenomenon has not yet been proven to compromise the short and mid-term effectiveness of cotrimoxazole prophylaxis [38]. Therefore, there are no compelling reasons to believe that our approach could be of any harm from a public health perspective. The rapid evolution of antifolate resistance observed in malaria in sub-Saharan African countries -where bacterial resistance to cotrimoxazole is higher and cross-resistance between cotrimoxazole and sulfadoxine-pyrimethamine (SP) may impair SP efficacy for malaria treatment- raises questions about the durability of this strategy. A careful resistance analysis to evaluate the emergence of resistance to the drug should be performed to tailor malaria prevention.

The overall cost of vector control, personal protection and prophylaxis in our setting was really limited: only $€ 2 /$ patient allow a net gain in survival with a reduction of death/discharge ratio from 0.76 to 0.26 . Surely, there is a higher cost of malaria in-patient care compared to prevention [5]. Malaria's economic impact is enormous [6] and the high direct cost for treating illness justifies efforts to improve coverage of preventive measures. Malaria preventive strategies targeting high risk groups -as severely ill TB patients- in high burden seasons -as the rainy season- could reduce diagnostic and treatment direct cost for families (where user fees are applied) and health services (where health care is provided without charge) while reducing mortality. 
Population's clinical characteristics, TB pattern and HIV prevalence were fairly constant along the three years of the intervention. Mortality during the first week of admission, and therefore not influenced by malaria prophylaxis, was also always above $28 \%$ suggesting that late presentation to the health center was a major factor for in-hospital death. Unfortunately, it is a common problem in TB facilities across Africa [39,40]. There was no effect on mortality by age in our group of patients, even if susceptibility to severe malaria is affected by age, but this could be due to the relative small proportion of children in our group.

No drop-outs were observed. This is a remarkable data considering the high drop-out rates in many TB programs in Africa [1]. The free access to diagnosis and to all components of treatment (pharmacological and nutritional) that was offered by the hospital in those years was surely a major determinant for good compliance. Repeated health education (once a week) performed by trained personnel was also attractive for patients (96-99\% of admitted patients attended each class) and reinforced sleeping under bed nets as well other healthy behaviors.

Our study has several limits. Firstly, it is an observational study and therefore it doesn't have the power of a randomized study. Nevertheless, even if it doesn't compare the three different strategies in a randomized fashion, the three-step intervention testes them subsequently and suggests the additive benefit of each successive one (death/discharge ratio dropped from 0.79 to 0.55 to 0.26 ). Secondly, malaria was not evaluated as a secondary outcome and therefore there is no direct demonstration that the reduction in mortality was due to the reduction in the number of both malaria episodes and malaria related mortality. Even so, all treatment protocols and main clinical characteristic of patient population remained fairly unchanged along the three years, and the only modifying interventions were related to malaria prevention. A reduction in positive blood films was indeed observed from the period August-November 2005 to AugustNovember 2006 and 2007 (23.65\% vs. 15.7\% vs. 8.4\% respectively, p-value 0.001 ), and even if the results were not registered on a regular basis and therefore are not completely reliable, they contribute to the hypothesis that malaria reduction was a determinant of reduced mortality. It is unlikely that patients died partly as a result of ineffective treatment for their malaria because chloroquine resistance is scarce in the country probably due to the higher chloroquine dosages routinely used [41].

\section{Conclusion}

In conclusion, our prospective observational study suggests that malaria vector control, personal protection and cotrimoxazole prophylaxis can be a safe and low cost measure to reduce in-hospital mortality in TB patients admitted in reference centers due to their poor clinical status and/or disseminated disease. Free care and health education have a role in reducing drop-outs in TB and malaria hospital-based programs. Prospective randomized trials are needed to confirm our findings and understand the role of cotrimoxazole in reducing overall morality and malaria related mortality in $\mathrm{TB}$ patients.

\section{Acknowledgements}

The Authors thank the Health Ministry of Guinea-Bissau and the Programa Nacional de Luta contra a Tuberculose for the support in TB drug and diagnostic reagents; USAID and the Global Fund for the support in mosquito nets, drugs and food to admitted patients; the Community of Sant'Egidio for rebuilding the hospital after the 1998-1999 civil war and for co-ordinating and sustaining the hospital's activity allowing all patients to receive free care.

\section{Author details}

'Clinic of Pediatric Hematology-Oncology, Department of Pediatrics, University of Padova, Padova, Italy. ${ }^{2}$ National Reference Hospital for Tuberculosis and Lung Disease, Bissau, Guinea- Bissau. ${ }^{3}$ Infectious Disease Unit, Department of Pediatrics, University of Padova, Padova, Italy. ${ }^{4}$ University of Padova, Padova, Italy. ${ }^{5}$ Department of Public Health and Cell Biology "Tor Vergata" University, Rome, Italy.

\section{Authors' contributions}

CR: designed the study, interpreted the data and wrote the manuscript; PM: interpreted the data and reviewed the manuscript; BF: designed the study and collected the data; VCS: performed the study and collected the data; ALA: performed the study and collected the data; VF: performed the study and collected the data; TS: performed statistical analysis; GC: reviewed the manuscript; RF: designed the study, interpreted the data and reviewed the manuscript.

All authors read and approved the final manuscript

\section{Competing interests}

The authors declare that they have no competing interests.

Received: 31 August 2010 Accepted: 2 March 2011

Published: 2 March 2011

\section{References}

1. World Health Organization: Global Tuberculosis control 2009: Epidemiology, strategy, financing. [http://www.who.int/tb/publications/global_report/ 2009/pdf/full_report.pdf], Last accessed 26 March 2010.

2. Soumaré M, Seydi M, Diop SA, Ba TA, Ndour CT, Dieng Y, Diop BM, Sow PS: The place of malaria in an infectious disease department in Dakar, Senegal. Med Trop (Mars) 2008, 68:485-90.

3. Petit PL, van Ginneken JK: Analysis of hospital records in four African countries, 1975-1990, with emphasis on infectious diseases. J Trop Med Hyg. 1995, 98:217-27.

4. Dgedge M, Novoa A, Macassa G, Sacarlal J, Black J, Michaud C, Cliff J: The burden of disease in Maputo City, Mozambique: registered and autopsied deaths in 1994. Bull World Health Organ. 2001, 79:546-52.

5. Hongoro C, McPake B: Hospital costs of high-burden diseases: malaria and pulmonary tuberculosis in a high HIV prevalence context in Zimbabwe. Trop Med Int Health. 2003, 8:242-50.

6. Russell S: The economic burden of illness for households in developing countries: a review of studies focusing on malaria, tuberculosis, and human immunodeficiency virus/acquired immunodeficiency syndrome. Am J Trop Med Hyg 2004, 71(2 Suppl):147-55.

7. Zachariah R, Spielmann MP, Harries AD, Salaniponi FM: Moderate to severe malnutrition in patients with tuberculosis is a risk factor associated with early death. Trans R Soc Trop Med Hyg. 2002, 96:291-4. 
8. van Lettow M, Fawzi WW, Semba RD: Triple trouble: the role of malnutrition in tuberculosis and human immunodeficiency virus coinfection. Nutr Rev 2003, 61:81-90.

9. Anya SE: Seasonal variation in the risk and causes of maternal death in the Gambia: malaria appears to be an important factor. Am J Trop Med Hyg. 2004, 70:510-3.

10. Romagosa C, Ordi J, Saute F, Quintó L, Machungo F, Ismail MR, Carrilho C Osman N, Alonso PL, Menendez C: Seasonal variations in maternal mortality in Maputo, Mozambique: the role of malaria. Trop Med Int Health. 2007, 12:62-7.

11. Ellner JJ: Immunoregulation in TB: observations and implications. Clin Trans/ Sci. 2010, 3:23-8.

12. Scott CP, Kumar N, Bishai WR, Manabe YC: Short report: modulation of Mycobacterium tuberculosis infection by Plasmodium in the murine model. Am J Trop Med Hyg. 2004, 70:144-8.

13. Hawkes M, Li X, Crockett M, Diassiti A, Conrad Liles W, Liu J, Kain KC: Malaria exacerbates experimental mycobacterial infection in vitro and in vivo. Microbes Infect 2010, 12:864-74.

14. Enwere GC, Ota MO, Obaro SK: The host response in malaria and depression of defence against tuberculosis. Ann Trop Med Parasitol. 1999, 93:669-78

15. Parry E, Godfrey R, Mabey D, Gill Geoffrey: Malaria in Principles of Medicine in Africa. Cambridge University Press; 2004

16. ter Kuile FO, Terlouw DJ, Phillips-Howard PA, Hawley WA, Friedman JF, Kolczak MS, Kariuki SK, Shi YP, Kwena AM, Vulule JM, Nahlen BL: Impact of permethrin-treated bed nets on malaria and all-cause morbidity in young children in an area of intense perennial malaria transmission in western Kenya: cross-sectional survey. Am J Trop Med Hyg 2003, 68(4 Suppl):100-7.

17. Lindblade KA, Eisele TP, Gimnig JE, Alaii JA, Odhiambo F, ter Kuile FO, Hawley WA, Wannemuehler KA, Phillips-Howard PA, Rosen DH, Nahlen BL, Terlouw DJ, Adazu K, Vulule JM, Slutsker L: Sustainability of reductions in malaria transmission and infant mortality in western Kenya with use of insecticide-treated bednets: 4 to 6 years of follow-up. JAMA. 2004 291:2571-80

18. Jaenson TG, Gomes MJ, Barreto dos Santos RC, Petrarca V, Fortini D, Evora J, Crato J: Control of endophagic Anopheles mosquitoes and human malaria in Guinea Bissau, West Africa by permethrin-treated bed nets. Trans R Soc Trop Med Hyg. 1994, 88:620-4.

19. Mermin J, Ekwaru JP, Liechty CA, Were W, Downing R, Ransom R, Weidle P, Lule J, Coutinho A, Solberg P: Effect of co-trimoxazole prophylaxis, antiretroviral therapy, and insecticide-treated bednets on the frequency of malaria in HIV-1-infected adults in Uganda: a prospective cohort study. Lancet. 2006, 367:1256-61.

20. Mabaso ML, Sharp B, Lengeler C: Historical review of malarial control in southern African with emphasis on the use of indoor residual housespraying. Trop Med Int Health. 2004, 9:846-56.

21. Grimwade K, Sturm AW, Nunn AJ, Mbatha D, Zungu D, Gilks CF: Effectiveness of cotrimoxazole prophylaxis on mortality in adults with tuberculosis in rural South Africa. AIDS. 2005, 19:163-8.

22. Wiktor SZ, Sassan-Morokro M, Grant AD, Abouya L, Karon JM, Maurice C, Djomand G, Ackah A, Domoua K, Kadio A, Yapi A, Combe P, Tossou O, Roels TH, Lackritz EM, Coulibaly D, De Cock KM, Coulibaly IM, Greenberg AE: Efficacy of trimethoprim-sulphamethoxazole prophylaxis to decrease morbidity and mortality in HIV-1-infected patients with tuberculosis in Abidjan, Côte d'Ivoire: a randomised controlled trial. Lancet 1999, 353:1469-75, Erratum in: Lancet 1999 Jun;353:2078.

23. Etard JF, Kodio B, Ronsmans C: Seasonal variation in direct obstetric mortality in rural Senegal: role of malaria? Am J Trop Med Hyg 2003, 68:503-4.

24. Cissé B, Sokhna C, Boulanger D, Milet J, Bâel H, Richardson K, Hallett R, Sutherland C, Simondon K, Simondon F, Alexander N, Gaye O, Targett G, Lines J, Greenwood B, Trape JF: Seasonal intermittent preventive treatment with artesunate and sulfadoxine-pyrimethamine for prevention of malaria in Senegalese children: a randomised, placebocontrolled, double-blind trial. Lancet. 2006, 367:659-67.

25. Plan: [http://www.plan-uk.org/wherewework/westafrica/guineabissau/ rollingbackmalaria/], Accessed 10 April 2010

26. World Health Organization: [http://www.who.int/malaria/publications/ country-profiles/mal2008-guinea-bissau-en.pdf], Accessed 10 April 2010.
27. Mermin J, Lule J, Ekwaru JP, Malamba S, Downing R, Ransom R, Kaharuza F, Culver D, Kizito F, Bunnell R, Kigozi A, Nakanjako D, Wafula W, Quick R: Effect of co-trimoxazole prophylaxis on morbidity, mortality, CD4-cell count, and viral load in HIV infection in rural Uganda. Lancet. 2004, 364:1428-34.

28. Kamya MR, Gasasira AF, Achan J, Mebrahtu T, Ruel T, Kekitiinwa A, Charlebois ED, Rosenthal PJ, Havlir D, Dorsey G: Effects of trimethoprimsulfamethoxazole and insecticide-treated bednets on malaria among HIV-infected Ugandan children. AIDS. 2007, 21:2059-66.

29. Brentlinger $P E$, Behrens $C B$, Micek MA: Challenges in the concurrent management of malaria and HIV in pregnancy in sub-Saharan Africa. Lancet Infect Dis 2006, 6:100-11.

30. Belay $\mathrm{M}$, Deressa $\mathrm{W}$ : Use of insecticide treated nets by pregnant women and associated factors in a pre-dominantly rural population in northern Ethiopia. Trop Med Int Health. 2008, 13:1303-13.

31. World Health Organization, Malaria Vector Control and Personal Protection, Report of WHO Study Group: WHO Technical Report Series 936. Geneva, Switzerland; 2004 [http://whqlibdoc.who.int/trs/WHO_TRS_936_eng.pdf], Last accessed 26 March 2010.

32. Protopopoff N, Van Bortel W, Marcotty T, Van Herp M, Maes P, Baza D, D'Alessandro U, Coosemans M: Spatial targeted vector control is able to reduce malaria prevalence in the highlands of Burundi. Am J Trop Med Hyg. 2008, 79:12-8.

33. Malamba SS, Mermin J, Reingold A, Lule JR, Downing R, Ransom R, Kigozi A, Hunt BM, Hubbard A, Rosenthal PJ, Dorsey G: Effect of cotrimoxazole prophylaxis taken by human immunodeficiency virus (HIV)-infected persons on the selection of sulfadoxine-pyrimethamineresistant malaria parasites among HIV-uninfected household members. Am J Trop Med Hyg. 2006, 75:375-80.

34. Thera MA, Sehdev PS, Coulibaly D, Traore K, Garba MN, Cissoko Y, Kone A, Guindo A, Dicko A, Beavogui AH, Djimde AA, Lyke KE, Diallo DA, Doumbo OK, Plowe CV: Impact of trimethoprim-sulfamethoxazole prophylaxis on falciparum malaria infection and disease. I Infect Dis. 2005, 192:1823-9.

35. Nunn AJ, Mwaba P, Chintu C, Mwinga A, Darbyshire JH, Zumla A: UNZAUCLMS Project LUCOT Collaboration: Role of co-trimoxazole prophylaxis in reducing mortality in HIV infected adults being treated for tuberculosis: randomised clinical trial. BMJ 2008, 10:337:a257.

36. Mulenga V, Ford D, Walker AS, Mwenya D, Mwansa J, Sinyinza F, Lishimpi K, Nunn A, Gillespie S, Zumla A, Chintu C, Gibb DM: CHAP Trial Team: Effect of cotrimoxazole on causes of death, hospital admissions and antibiotic use in HIV-infected children. AIDS 2007, 21(1):77-84

37. Baden LR, Katz JT, Franck L, Tsang S, Hall M, Rubin RH, Jarcho J: Successful toxoplasmosis prophylaxis after orthotopic cardiac transplantation with trimethoprim-sulfamethoxazole. Transplantation 2003, 75(3):339-43.

38. Zachariah R, Harries AD, Spielmann MP, Arendt V, Nchingula D, Mwenda R, Courtielle O, Kirpach P, Mwale B, Salaniponi FM: Changes in Escherichia coli resistance to co-trimoxazole in tuberculosis patients and in relation to co-trimoxazole prophylaxis in Thyolo, Malawi. Trans R Soc Trop Med Hyg 2002, 96:202-4

39. Harries AD, Hargreaves NJ, Gausi F, Kwanjana JH, Salaniponi FM: High early death rate in tuberculosis patients in Malawi. Int J Tuberc Lung Dis. 2001, 5:1000-5.

40. Sacks LV, Pendle S: Factors related to in-hospital deaths in patients with tuberculosis. Arch Intern Med 1998, 158:1916-22.

41. Ursing J, Kofoed PE, Rodrigues A, Rombo L: No seasonal accumulation of resistant $\mathrm{P}$. falciparum when high-dose chloroquine is used. PLoS One 2009, 4(8):e6866.

Pre-publication history

The pre-publication history for this paper can be accessed here: http://www.biomedcentral.com/1471-2334/11/57/prepub

doi:10.1186/1471-2334-11-57

Cite this article as: Colombatti et al:: Malaria prevention reduces inhospital mortality among severely ill tuberculosis patients: a three-step intervention in Bissau, Guinea-Bissau. BMC Infectious Diseases 2011 11:57. 